\title{
Optimization of Extraction Technology of Gynostemma Polysaccharides by Response Surface Methodology
}

\author{
Xiaodan Tang ${ }^{1, a}$, Junchen Song, Bo Li, Huan Lu, zhuo zhang \\ ${ }^{1}$ School of Chemical Engineering, University of Science and Technology Liaoning, \\ 185 Qianshan Zhong Road, Anshan, Liaoning, 114051, China \\ atxd413@163.com
}

Keywords: Gynostemma polysaccharides, response surface methodology, Box-Behnken design

\begin{abstract}
The aim of this work was to employ optimization strategy based on statistical experimental designs to enhance the polysaccharides extraction yield from Gynostemma pentaphyllum. The effects of three independent variables on the polysaccharides extraction yield were investigated and the optimal conditions were evaluated by means of Box-Behnken design. The optimal conditions are as follows: ratio of water to raw material 31 , extraction temperature $90^{\circ} \mathrm{C}$ and extraction time $2.5 \mathrm{~h}$. Under these conditions, the polysaccharides yield is $3.80 \pm 0.15 \%(N=5)$, which is agreed closely with the predicted yield value.
\end{abstract}

\section{Introduction}

Gynostemma pentaphyllum(Thunb.) Makino, a perennial liana herb belonging to the Cucurbitaceae, is widely distributed in China, especially the south provinces of the Yangtze River and Qinling Mountains, Japan, Korea and Southeast Asia countries. Phytochemical studies on this plant have identified it is a saponin-rich plant which is closely related to the component saponins in expensive ginseng, and be regarded as "second ginseng"[1]. Recently, polysaccharides obtained from G. pentaphyllum has attracted greater attention owing to its antitumor activities, anti-gastric ulcer effect, immunomodulatory effect, anti-aging, antioxidant properties and treating hyperlipidemia $^{[2]}$. However, there has not been much study on the extraction optimization of polysaccharides from $G$. pentaphyllum.

Response Surface Methodology(RSM) is an affective mathematical and statistical techniques , which apply multivariate quadratic regression to fit the functional relationship between factors and response values and analy the regression equation to find the optimal process parameters. The main advantage of RSM is the reduced number of experimental trials needed to evaluate multiple parameters and their interactions. Therefore, it is less laborious and time-consuming than other approaches required to optimize a process ${ }^{[3]}$..

In this study, the main objective was to optimize the extraction parameters of polysaccharides from G. pentaphyllum using RSM and employ a Box-Behnken design to study the effects of ratio of water to raw material, extraction temperature and extraction time on the polysaccharides yield and their interactions.

\section{Experimental}

Materials and instrument. Dried G. pentaphyllum was purchased from Guang Ming Prepared Medicinal Herbs Factory (Hebei, China). Glucose $\left(\mathrm{C}_{6} \mathrm{H}_{12} \mathrm{O}_{6}\right)$ as standard was purchased from Guoyao Chemical Reagent Co. (Shenyang, China). All other chemicals were of analytical grade. The analysis of gynostemma polysaccharides was carried out on a 722 spectrometry from Shanghai YuLong instrument Co., LTD.

Methods. The G. pentaphyllum was extracted with $95 \%$ ethanol at $50^{\circ} \mathrm{C}$ for $2 \mathrm{~h}$ for 2 times and then the residues were dried. A $10 \mathrm{~g}$ dried residues of $G$. pentaphyllum was extracted in water bath with distilled water in a designed ratio of water to raw material, extraction temperature and extraction time. The supernatant was collected for the determination of polysaccharides yield. 
Determination. Total glucose concentration was measured by phenol-vitriol method using glucose as standard. For quantization, standard solutions of $15 \mu \mathrm{g} / \mathrm{mL}$ to $180 \mu \mathrm{g} / \mathrm{mL}$ were prepared separately by transferring $(0.5$ to $6 \mathrm{~mL})$ of stock solution $(0.3 \mathrm{mg} / \mathrm{mL})$ to $10 \mathrm{~mL}$ volumetric flasks and adjusted the volume with distilled water. Quantification was carried out by the absorbance using external standard method. Each absorbance value of the standard and the sample solution was recorded by a 722 spectrophotometer at the wavelength of $490 \mathrm{~nm}$. At the same time, the solution was measured as blank control in an identical way. The standard curve was obtained by plotting concentration ratio against its absorbance ratio, and the regression equations was $A=0.0107 C-0.1221$ with $R^{2}$ being 0.992. The polysaccharides content of sample extract was calculated by regress equation.

Design of statistical experiment. On the basis of single-factor experiment, Box-Behnken design was employed for experimental design, data analysis and model building with software Design Expert(Trial Version 7.0.0, Stat-Ease, Inc, Minneapolis, USA). Three independent variables used in this work were ratio of water to raw material $\left(X_{1}\right)$, extraction temperature $\left(X_{2}\right)$ and extraction time $\left(X_{3}\right)$, with three levels for each variable, while the dependent variable was the polysaccharides yield. The symbols and levels are shown in Table 1.The whole design consisted of 17 experimental points carried out in random order. Based on BBD data, regression analysis was performed and was fitted into a quadratic polynomial model:

$$
Y=\beta_{0}+\sum_{i=1}^{3} \beta_{i} X_{i}+\sum_{i=1}^{3} \beta_{i i} X_{i}^{2}+\sum_{i=1}^{2} \sum_{j=i+1}^{3} \beta_{i j} X_{i} X_{j}
$$

Where $Y$ is the response function; $\beta_{0}, \beta_{i}, \beta_{i i}$ and $\beta_{i j}$ represent the constant regression coefficients; $X_{i}$ and $X_{j}$ are the coded independent variables.

\section{Results and discussion}

Statistical analysis and the model fitting. The mathematical model describing the extraction yield of polysaccharides as a function of the coded independent variables in the selected ranges was given by the following equation:

$$
\begin{array}{rr}
R_{1}=3.75+0.32 X_{1}-0.070 X_{2}+0.12 \times C-0.045 X_{1} X_{2}+0.25 X_{1} X_{3} \\
X_{2}{ }^{2}-0.21 \times X_{3}{ }^{2} & +0.100 X_{2} X_{3}-0.58 \times X_{1}^{2}-0.32
\end{array}
$$

The experimental data were statistically analyzed by Design Expert software for analysis of variance (ANOVA) and the results were shown in Table 2. The value of determination coefficients $R^{2}(0.9955)$ for Eq.2 indicates a high degree of correlation between the observed and predicted values, only $0.45 \%$ of the total variations are not explained by the model. The value of the adjusted determination coefficient $\left(R_{\text {Adj }}^{2}=0.9898\right)$, which also proves that the regression model defined well the true behavior of the system. At the same time, a relatively lower value of the coefficient of variation $(C V=1.46 \%)$ indicates a better precision and reliability of the experiments values. 
Table 1 Box-Behnken design and the response for the polysaccharides extraction yield

\begin{tabular}{ccccc}
\hline Run & $\begin{array}{c}X_{1} \text { (ratio of water to raw } \\
\text { material) }\end{array}$ & $\begin{array}{c}X_{2} \text { (extraction } \\
\left.\text { temperature, }{ }^{\circ} \mathrm{C}\right)\end{array}$ & $\begin{array}{c}X_{3}(\text { extraction } \\
\text { time, min) }\end{array}$ & $\begin{array}{c}\text { Extraction yield } \\
(\%)\end{array}$ \\
\hline 1 & $0(1: 30)$ & $0(90)$ & $0(2)$ & 3.73 \\
2 & $0(1: 30)$ & $0(90)$ & $0(2)$ & 3.74 \\
3 & $-1(1: 25)$ & $0(90)$ & $1(3)$ & 2.48 \\
4 & $0(1: 30)$ & $0(90)$ & $0(2)$ & 3.72 \\
5 & $0(1: 30)$ & $1(100)$ & $-1(1)$ & 2.89 \\
6 & $0(1: 30)$ & $0(90)$ & $0(2)$ & 3.78 \\
7 & $0(1: 30)$ & $1(100)$ & $1(3)$ & 3.35 \\
8 & $0(1: 30)$ & $-1(80)$ & $1(3)$ & 3.36 \\
9 & $1(1: 35)$ & $1(100)$ & $0(2)$ & 3.08 \\
10 & $0(1: 30)$ & $0(90)$ & $0(2)$ & 3.77 \\
11 & $1(1: 35)$ & $-1(80)$ & $0(2)$ & 3.24 \\
12 & $0(1: 30)$ & $-1(80)$ & $-1(1)$ & 3.30 \\
13 & $1(1: 35)$ & $0(90)$ & $1(3)$ & 3.65 \\
14 & $-1(1: 25)$ & $-1(80)$ & $0(2)$ & 2.54 \\
15 & $-1(1: 25)$ & $1(100)$ & $0(2)$ & 2.56 \\
16 & $1(1: 35)$ & $0(90)$ & $-1(1)$ & 2.94 \\
17 & $-1(1: 25)$ & $0(90)$ & $-1(1)$ & 2.78 \\
\hline
\end{tabular}

In this experiment, the $P$-value of the model was less than 0.0001 . Meanwhile, the lack of fit value of the model was 0.0527 which was not significant. These two values confirm that the model equation is adequate for predicting the polysaccharides yield within the range of experimental variables. The significance of each coefficient of Eq. 2 was checked using $P$-value. If the $P$-value becomes smaller, the corresponding coefficient would be more significant ${ }^{[4]}$. The data in Table 2 indicated that the variables with the largest effect were the linear coefficient $X_{1}, X_{3}$, the interaction coefficient $X_{1} X_{3}$ and the quadratic coefficient $X_{1}{ }^{2}, X_{2}{ }^{2}, X_{3}{ }^{2}(P<0.001)$. Besides, the linear coefficient $X_{2}$ and the interaction coefficient $X_{2} X_{3}$ were also found significant $(P<0.01)$.

Table 2 Regression coefficients and analysis of the predicted quadratic polynomial model

\begin{tabular}{cccccc}
\hline Soruce & Sum of Squares & df & Mean Square & $F$ value & $\begin{array}{c}P \text {-value } \\
\text { Prob }>\text { F }\end{array}$ \\
\hline Model & 3.45 & 9 & 0.38 & 173.25 & $<0.0001^{\mathrm{a}}$ \\
$X_{1}-X_{1}$ & 0.81 & 1 & 0.81 & 366.96 & $<0.0001^{\mathrm{a}}$ \\
$X_{2}-X_{2}$ & 0.039 & 1 & 0.039 & 17.70 & $0.0040^{\mathrm{b}}$ \\
$X_{3}-X_{3}$ & 0.11 & 1 & 0.11 & 48.81 & $0.0002^{\mathrm{a}}$ \\
$X_{1} X_{2}$ & $8.100 \times 10^{-3}$ & 1 & $8.100 \times 10^{-3}$ & 3.66 & $0.0974^{\mathrm{c}}$ \\
$X_{1} X_{3}$ & 0.26 & 1 & 0.26 & 115.14 & $<0.0001^{\mathrm{a}}$ \\
$X_{2} X_{3}$ & 0.040 & 1 & 0.040 & 18.06 & $0.0038^{\mathrm{b}}$ \\
$X_{1}{ }^{2}$ & 1.41 & 1 & 1.41 & 634.52 & $<0.0001^{\mathrm{a}}$ \\
$X_{2}{ }^{2}$ & 0.42 & 1 & 0.42 & 188.92 & $<0.0001^{\mathrm{a}}$ \\
$X_{3}{ }^{2}$ & 0.18 & 1 & 0.18 & 82.04 & $<0.0001^{\mathrm{a}}$ \\
Residual & 0.016 & 7 & $2.215 \times 10^{-3}$ & - & \\
Lack of fit & 0.013 & 3 & $4.275 \times 10^{-3}$ & 6.38 & $0.0527^{\mathrm{c}}$ \\
Pure Error & $2.680 \times 10^{-3}$ & 4 & $6.700 \times 10^{-4}$ & - & \\
Cor Total & 3.47 & 16 & & - & \\
\hline
\end{tabular}

${ }^{\mathrm{a}}$ Means significance $(P<0.001) ;{ }^{\mathrm{b}}$ Means significance $(P<0.01) ;{ }^{\mathrm{c}}$ Not significant

Effect of extraction variables on the polysaccharides yield. In this study, the 3D response surface plots are presented in Fig.1.Two variables within the experimental range were depicted in one 3D surface plot while the other variable was fixed constant at zero level. Different shapes of the contour plots indicate different interactions between the variables. Elliptical contours are obtained when there is a perfect interaction between the independent variables while circular contour plot 
indicates otherwise ${ }^{[5]}$. As shown in Fig.1(a), the polysaccharides extraction yield was increased with increasing of ratio of water to raw material from 25 to 31.5, but beyond 31.5, extraction yield decreased with increasing ratio of water to raw material. From Fig.1(b), it can be seen that maximum extraction yield can be achieved when ratio of water to raw material and extraction time were 31.5 and $2.5 \mathrm{~h}$ respectively. The polysaccharides extraction yield increased evidently with increasing of extraction time from 1 to 2.5, but beyond 2.5h, the extraction yield descended. The 3D response surface plot based on independent variables extraction time and extraction temperature were shown in Fig. 1(C). An increase in polysaccharides yield could be significantly achieved with the increases of extraction time. It was obvious that the polysaccharides yield was increased with the increasing extraction temperature from 80 to $88.3{ }^{\circ} \mathrm{C}$, meaning that further increases of extraction temperature would decrease the yield.
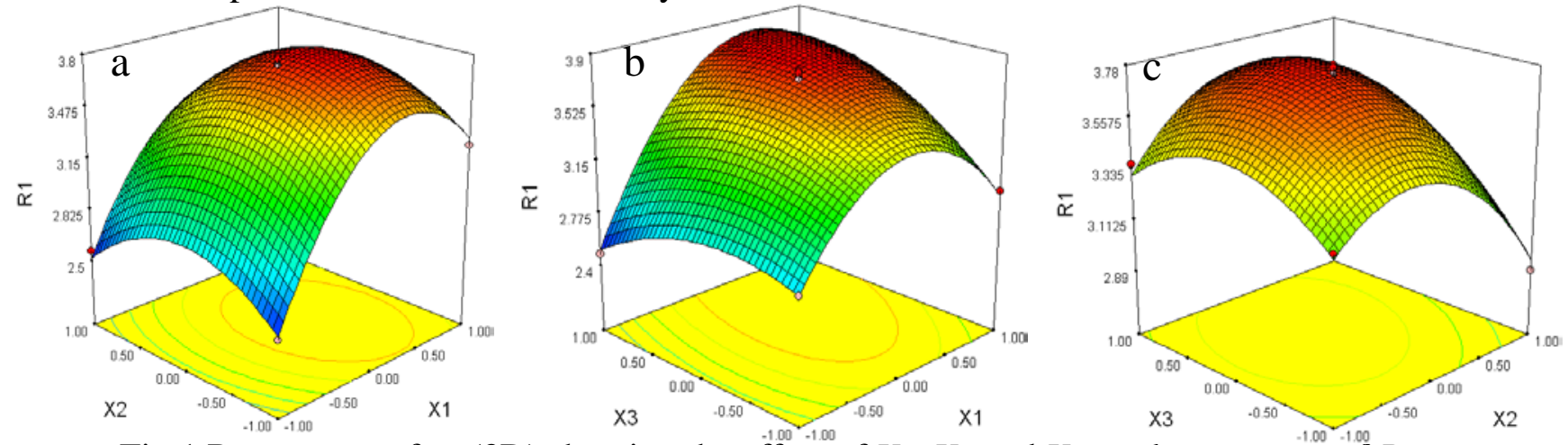

Fig.1 Response surface(3D) showing the effect of $X_{1}, X_{2}$ and $X_{3}$ on the response of $R_{1}$

Validation of the model. According to Fig.1, the optimal conditions were ratio of water to raw material 31.5 , extraction temperature $88.3^{\circ} \mathrm{C}$, extraction time $2.5 \mathrm{~h}$ and the model predicted maximum response of $3.83 \%$. To ensure the predicted result was not biased toward the practical value, experiment rechecking was performed using this modified optimal conditions: ratio of water to raw material of 31, extraction temperature of $90^{\circ} \mathrm{C}$ and extraction time of $2.5 \mathrm{~h}$. After extraction under these optimal conditions, the polysaccharides yield is $3.80 \pm 0.15 \%(N=5)$, which is in agreement with the predicted value significantly. The results of analysis confirm that the regression model is satisfactory and accurate.

\section{Conclusions}

The response surface methodology was successfully employed to optimize the polysaccharides extraction from Gynostemma pentaphyllum Makino. Using Box-Behnken design for the three selected ingredients, the interactions between the components and their optimum levels for maximum polysaccharides extraction were determined. The experimentally found value of polysaccharides extraction using statistically designed medium was in perfect agreement with the predicted value. The results in this work can be useful to the development of industrial extraction processes.

\section{References}

[1] Lv, Y., Yang, X.. B.,Zhao, y., Ruan, Y., Yang, Y.,Wang, Z. Z. Separation and quantification of component monosaccharides of the tea polysaccharides from Gynostemma pentaphyllum by HPLC with indirect UV detection. Food Chemistry, 2009, 112, 742-746.

[2] Z. J. Wang, D. H. Luo: Carbohydrate Polymers. Antioxidant activities of different fractions of polysaccharide purified from Gynostemma pentaphyllum Makino[J]. 2007, 68, 54-65.

[3] Box, G. E. P., Behnken, D. W. Some new three level designs for the study of quantitative variables[J]. Technometrics,1960, 2, 455-475.

[4] Gan, C. Y., Latiff, A. A. Extraction of antioxidant pectic-polysaccharide from mangosteen (Garcinia mangostana) rind: Optimization using response surface methodology[J]. Carbohydrate 
Polymers, 2011, 83, 600-607.

[5] Guo, X., Zou, X., Sun,M. Optimization of extraction process by response surface methodology and preliminary characterization of polysaccharides from Phellinus igniarius[J]. Carbohydrate Polymers, 2010, 80,344-349. 